\title{
A PCR-RFLP method for genotyping of inversion 2Rc in Anopheles coluzzii
}

\author{
Raquel Montanez-Gonzalez ${ }^{1,2 \dagger}$, Alexandra C. Vallera ${ }^{1 \dagger}$, Maria Calzetta ${ }^{3}$, Verena Pichler ${ }^{3}$, Rachel R. Love ${ }^{1,2}$, \\ Moussa W. Guelbeogo ${ }^{4}$, Roch K. Dabire ${ }^{5}$, Marco Pombi ${ }^{3}$, Carlo Costantini ${ }^{6}$, Frederic Simard ${ }^{6}$, \\ Alessandra della Torre ${ }^{3}$ and Nora J. Besansky ${ }^{1,2^{*}}$ (1)
}

\begin{abstract}
Background: Genotyping of polymorphic chromosomal inversions in malaria vectors such as An. coluzzii Coetzee \& Wilkerson is important, both because they cause cryptic population structure that can mislead vector analysis and control and because they influence epidemiologically relevant eco-phenotypes. The conventional cytogenetic method of genotyping is an impediment because it is labor intensive, requires specialized training, and can be applied only to one gender and developmental stage. Here, we circumvent these limitations by developing a simple and rapid molecular method of genotyping inversion 2Rc in An. coluzzii that is both economical and field-friendly. This inversion is strongly implicated in temporal and spatial adaptations to climatic and ecological variation, particularly aridity.

Methods: Using a set of tag single-nucleotide polymorphisms (SNPs) strongly correlated with inversion orientation, we identified those that overlapped restriction enzyme recognition sites and developed four polymerase chain reaction (PCR) restriction fragment length polymorphism (RFLP) assays that distinguish alternative allelic states at the tag SNPs. We assessed the performance of these assays using mosquito population samples from Burkina Faso that had been cytogenetically karyotyped as well as genotyped, using two complementary high-throughput molecular methods based on tag SNPs. Further validation was performed using mosquito population samples from additional West African (Benin, Mali, Senegal) and Central African (Cameroon) countries.
\end{abstract}

Results: Of four assays tested, two were concordant with the 2Rc cytogenetic karyotype $>90 \%$ of the time in all samples. We recommend that these two assays be employed in tandem for reliable genotyping. By accepting only those genotypic assignments where both assays agree, > 99\% of assignments are expected to be accurate.

Conclusions: We have developed tandem PCR-RFLP assays for the accurate genotyping of inversion 2Rc in An. coluzzii. Because this approach is simple, inexpensive, and requires only basic molecular biology equipment, it is widely accessible. These provide a crucial tool for probing the molecular basis of eco-phenotypes relevant to malaria epidemiology and vector control.

Keywords: Anopheles gambiae complex, Chromosomal inversion, Inversion genotyping, Malaria vector, Molecular karyotyping, PCR-RFLP, Tag SNP

\footnotetext{
*Correspondence: nbesansk@nd.edu

${ }^{\dagger}$ Raquel Montanez-Gonzalez and Alexandra C. Vallera contributed equally to this work

${ }^{1}$ Department of Biological Sciences, University of Notre Dame, Notre Dame, IN 46556, USA

Full list of author information is available at the end of the article
}

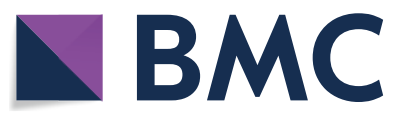

(c) The Author(s) 2021. This article is licensed under a Creative Commons Attribution 4.0 International License, which permits use, sharing, adaptation, distribution and reproduction in any medium or format, as long as you give appropriate credit to the original author(s) and the source, provide a link to the Creative Commons licence, and indicate if changes were made. The images or other third party material in this article are included in the article's Creative Commons licence, unless indicated otherwise in a credit line to the material. If material is not included in the article's Creative Commons licence and your intended use is not permitted by statutory regulation or exceeds the permitted use, you will need to obtain permission directly from the copyright holder. To view a copy of this licence, visit http://creativeco mmons.org/licenses/by/4.0/. The Creative Commons Public Domain Dedication waiver (http://creativecommons.org/publicdomain/ zero/1.0/) applies to the data made available in this article, unless otherwise stated in a credit line to the data. 


\section{Background}

Chromosomal inversions are taxonomically ubiquitous structural rearrangements resulting from the breakage and end-to-end reversal of a chromosome segment [1]. Growing numbers of studies powered by genomic sequencing suggest that inversions play important roles in sex chromosome evolution, speciation, and environmental adaptation, primarily because they suppress recombination between the inverted and corresponding non-inverted regions in heterozygotes [1-3]. Adaptive allelic combinations between inversion breakpoints are preserved as haplotype blocks against recombination with other genetic backgrounds. Balancing selection acting on environmentally adaptive variation appears to be a major force responsible for the maintenance of inversions and their involvement in local adaptation [1-4]. In most cases, however, the specific targets of selection within inversions and underlying molecular mechanisms controlling alternative phenotypes remain unknown.

The Afrotropical Anopheles gambiae complex radiated within the last 0.5 million years [5] into at least nine morphologically identical species [6-9], three of which are major vectors of human malaria. Across the complex, more than 120 inversion polymorphisms have been detected in natural populations, and an additional ten inversions are fixed between species [10]. Of note, the central part of chromosome $2 \mathrm{R}$ that overlaps the $2 \mathrm{R} c$ inversion in three taxa [An. gambiae (s.s.) (henceforth, An. gambiae), An. coluzzii, and An. arabiensis] is disproportionately involved in both fixed and polymorphic inversions in the species complex, potentially implicating the $2 \mathrm{R} c$ region in oviposition site specializations that distinguish taxa in this group [10]. Historically, fixed inversions provided crucial taxonomic tools for identification of these isomorphic species, while analysis of inversion polymorphisms such as $2 \mathrm{R} c$ segregating nonrandomly within species led to the recognition of assortatively mating 'chromosomal forms' presumed to be in the incipient stages of ecological speciation. One of these, the MOPTI chromosomal form (previously considered an incipient species of An. gambiae but now regarded as an arid-associated ecotype of An. coluzzii $[6,10])$, was characterized in Mali by three main alternative whole-arm karyotypic arrangements: $2 \mathrm{R} b c, 2 \mathrm{R} u$, and $2 \mathrm{R}+[11]$. Importantly, the $b c$ karyotypic arrangement (i.e. co-segregation of the adjacent $2 \mathrm{R} b$ and $2 \mathrm{R} c$ inversions on one arm) was found to be significantly correlated with climatic and ecological variation [11], as $b c$ regularly increased in frequency both in the dry season and in dry geographic localities (Sahelian and Saharan). Mosquito carriers of the $b c$ arrangement escape rain dependence through successful exploitation of irrigated sites for larval development and adult tolerance of extreme aridity. In an extensive review of these findings, the authors conclude: "The evidence for consistent temporal and spatial adaptive changes in $(2 \mathrm{R} b c)$ inversion frequencies is unquestionable" [p.505, ref. 11].

Until the advent of molecular approaches, cytotaxonomy was the only practical method for distinguishing species of An. gambiae (s.l.), ensuring the preservation of cytogenetic karyotyping skills despite the drawbacks. Cytogenetic karyotyping is labor-intensive, requires specialized training, and is applicable only to properly preserved ovarian tissue from adult An. gambiae (s.l.) females at the half-gravid gonotrophic stage. The development of an rDNA-based PCR diagnostic assay beginning in 1993 [12] eliminated the requirement for cytotaxonomy and led to declining cytogenetic expertise, especially after the rDNA assay was extended to $A n$. coluzzii (formerly M-molecular form, including MOPTI karyotypic arrangements) and An. gambiae (formerly S-molecular form, including mainly SAVANNA arrangements) [13].

Cytogenetic karyotyping had another important application in addition to species identification. Until recently, it was the only available tool for genotyping of inversion polymorphisms known to be significant predictors of epidemiologically relevant eco-phenotypes in An. gambiae (s.l.). As inversion polymorphism is a form of cryptic population substructure, unrecognized heterogeneities in population samples can bias genome-wide association studies as well as vector surveillance and control [14]. To address the need for an inversion genotyping method that does not rely on cytogenetics, we have been developing genomic approaches to identify and detect tag single nucleotide polymorphisms (SNPs) that are highly predictive of inversion orientation [15-17]. In addition to high-throughput molecular and in silico methods that can comprehensively genotype multiple tags from multiple inversions in parallel [15-17], we have also designed more conventional PCR-RFLP assays for individual inversions that, because they do not require expensive equipment or services, are both field- and budget-friendly [18]. Here, we complement our recently developed PCR-RFLP assays for inversion $2 \mathrm{R} b$ in An. gambiae and An. coluzzii [18] with assays for $2 \mathrm{R} c$ in An. coluzzii.

\section{Methods}

\section{Mosquito sampling and processing}

Anopheles coluzzii mosquitoes used in this study were from various historical Afrotropical collections motivated by polytene chromosome analysis of An. gambiae (s.l.). We focused our primary effort on an An. coluzzii population sampled from Burkina Faso, which lies in the arid Sudan savanna belt of West Africa. This geographic 
region was chosen because local An. coluzzii populations are characterized by high $2 \mathrm{R} c$ chromosomal inversion polymorphism [19]. Furthermore, the particular Burkina Faso population sample analyzed in this study $(n=463$; Additional file 1: Table S1) had been previously genotyped for chromosomal inversions using each of three independent methodologies that collectively provide a robust basis for evaluating performance of individual PCR-RFLP assays developed here: (i) classical cytogenetics based on phase contrast microscopy and two molecular approaches, (ii) amplicon sequencing (GT-Seq) and (iii) array hybridization (TaqMan Open Array), based on the detection of tag single nucleotide polymorphisms (SNPs) strongly correlated with inversion orientation [15].

Additional geographic population samples of cytogenetically karyotyped $A n$. coluzzii were obtained from three West African countries (Mali, $n=131$ [11]; Senegal, $n=2$ [20]; Benin, $n=20$ [21]) and one Central African country (Cameroon, $n=43$ [22]) (Additional file 1: Table S1).

Molecular identification to species DNA individually extracted by one of various methods was used as template in a PCR reaction for An. coluzzii species identification, based on the SINE200 assay [23] or ribosomal DNA (rDNA) [24, 25].

Inversion genotyping via cytogenetics and multilocus tags Polytene chromosome preparations from preserved ovarian nurse cells followed della Torre [26]. Paracentric inversion karyotypes were scored according to the $A n$. gambiae cytogenetic map [10, 27, 28] and established nomenclature [11]. High-throughput molecular inversion genotyping based on the simultaneous detection of multiple tag SNPs was conducted previously via amplicon sequencing or probe hybridization to arrays, as detailed in Love et al. [15].

\section{Assay design for PCR-RFLP genotyping of An. coluzzii 2Rc}

Tag SNPs predictive of $2 \mathrm{R} c$ genotype were computationally identified previously $[15,16]$ in the Ag1000G database of natural genomic variation [29, 30], a database constructed from deeply sequenced wild-caught individual An. gambiae and An. coluzzii mosquitoes. At the time of our tag SNP ascertainment, population samples of An. coluzzii represented in Ag1000G came from Angola, Burkina Faso, Cameroon, Cote d'Ivoire, Ghana, Guinea, and Mali (we omitted samples from The Gambia and Guinea Bissau that were admixed with An. gambiae), and only a subset of those from Burkina Faso, Cameroon, and Mali carried metadata about cytogenetic karyotype. Accordingly, tag SNPs for An. coluzzii 2Rc were identified in the pooled Burkina Faso and Angola Ag1000G samples following Ma and Amos [31], who showed that the application of principal components analysis (PCA) to SNP genotypes within the local window of the genome containing an inverted region produces a pattern indicative of two distinct "populations" of inversion homozygotes (inverted and standard) and their 1:1 admixture (inversion heterozygotes), a pattern of population substructure created by suppressed recombination in the inverted region.

Briefly, to apply this approach, we created a matrix of one-digit genotypes at biallelic SNPs within $2 \mathrm{R} c$ for each mosquito. One-digit SNP genotypes represent the count $(0,1$, or 2$)$ of alternate alleles (i.e. variants with respect to the PEST reference sequence at a SNP position). PCA of the resulting data matrix allowed computational imputation of the $2 R c$ inversion genotype for mosquitoes in the sample. Individual SNPs capable of accurately predicting inversion genotype (tag SNPs) should have allelic states that are strongly correlated with inversion genotype. The correlation at individual candidate tags was measured as the percentage of the total mosquito sample with matching inversion and SNP genotypes. To be conservative, we calculated the correlation separately for each of the three inversion genotypes, adopting the minimum value across the three genotypes as the final genotypic concordance value. Candidate tags were ranked based on the final genotypic concordance values.

Unlike high-throughput molecular inversion genotyping based on tens of tags per inversion, conventional PCR-RFLP genotyping necessarily relies on one or a few tags, each of which should thus have the highest genotypic concordance. Although we prioritized such tags, suitable candidates for RFLP also depend on the serendipitous overlap of the tag SNP with the recognition site of a commercially available restriction enzyme, such that recognition and cleavage depend upon allelic status of the tag on both chromosomes in a diploid mosquito. Additional constraints include the ability to design suitable flanking PCR primers (e.g. free of possible hairpin or primer-primer interactions), which anneal to high-complexity, non-repetitive template DNA to reduce off-target mis-priming. Applying a minimum threshold of $85 \%$ genotypic concordance to the ranked tags, we screened the qualified tags for those that overlapped restriction enzyme recognition sites, using NEBcutter v2.0 [32]. Using the An. gambiae PEST reference genome accessed through VectorBase [33] and Primer3Plus v2.4.2 [34], we designed primer pairs that flanked each tag SNP and produced amplicons 200-300 bp in length. We avoided any primer binding sites and restriction enzyme recognition sites that contained high frequency variants $(>5 \%$, as judged from Ag1000G variation data) and also excluded primer binding sites that overlapped repetitive sequence (as judged from softmasking of AgamP4). Assays whose 
electrophoretic profiles provided the best separation between inversion genotypes were prioritized.

\section{PCR-RFLP genotyping}

PCR was carried out in $25 \mu \mathrm{l}$ reactions containing $20 \mathrm{mM}$ Tris- $\mathrm{HCl}(\mathrm{pH} 8.3), 50 \mathrm{mM} \mathrm{KCl}, 200 \mu \mathrm{M}$ of each dNTP, $2 \mathrm{mM} \mathrm{MgCl}, 10$ pmol of each primer, $1 \mathrm{U}$ of Taq polymerase, and $1 \mu \mathrm{l}$ of template genomic DNA. PCR conditions included an initial incubation at $94{ }^{\circ} \mathrm{C}$ for $2 \mathrm{~min}, 35$ cycles of $94{ }^{\circ} \mathrm{C}$ for $30 \mathrm{~s}, 57^{\circ} \mathrm{C}$ for $30 \mathrm{~s}$, and $72{ }^{\circ} \mathrm{C}$ for $45 \mathrm{~s}$, followed by $72{ }^{\circ} \mathrm{C}$ for 2 min and a $4{ }^{\circ} \mathrm{C}$ hold. PCR amplification was confirmed via gel electrophoresis on $1 \%$ agarose gels stained with SYBR Safe at $135 \mathrm{~V}$ in $0.5 \times \mathrm{TBE}$ buffer.

An $8 \mu \mathrm{l}$ aliquot of the resulting PCR product was digested in $20 \mu \mathrm{l}$ reactions with $0.5 \mu \mathrm{l}$ restriction enzyme and $1 \times$ Cutsmart buffer following the recommendations of the manufacturer (New England Biolabs, Ipswich, MA, USA): HinfI and HaeII reactions were incubated at $37^{\circ} \mathrm{C}$ for $1 \mathrm{~h}$, then heat inactivated at $80^{\circ} \mathrm{C}$ for $20 \mathrm{~min}$; Cac8I incubated at $37^{\circ} \mathrm{C}$ for $1 \mathrm{~h}$, then heat inactivated at $65^{\circ} \mathrm{C}$ for $20 \mathrm{~min}$; BstUI incubated at $60^{\circ} \mathrm{C}$ for $1 \mathrm{~h}$ without heat inactivation. Optionally, SDS loading dye was prepared with $10 \mu \mathrm{l}$ of $10 \%$ SDS and $1 \mathrm{ml}$ of $6 \times$ loading dye to mitigate protein-DNA interactions and improve band quality. Digest products were analyzed via gel electrophoresis on $2.5-3 \%$ agarose gels stained with SYBR Safe at $100 \mathrm{~V}$ in $0.5 \times$ TBE buffer.

\section{Amplicon sequencing}

Enzymatic cleanup of the amplified PCR product was achieved in reactions containing $2 \mathrm{U}$ of Exonuclease 1 (USB Corporation, Cleveland, OH), 1U of Shrimp Alkaline Phosphate (USB), $1.8 \mu \mathrm{l}$ of $\mathrm{ddH}_{2} \mathrm{O}$, and $8 \mu \mathrm{l}$ of the PCR product. After incubation at $37^{\circ} \mathrm{C}$ for $15 \mathrm{~min}$, the enzymes were inactivated at $80{ }^{\circ} \mathrm{C}$ for $15 \mathrm{~min}$. Sanger sequencing was performed directly on the resulting samples, using the forward PCR primer (Table 1) and the ABI 3730X1 DNA Analyzer Platform (ThermoFisher Scientific, Waltham, MA), by the Genomics and Bioinformatics Core Facility at the University of Notre Dame or by Eurofins Genomics Italy. Sequences were deposited with GenBank under accessions MW158473-MW158543.

\section{Results and discussion}

Four candidate tag SNPs met the four design criteria for PCR-RFLP assays (see"Methods"): (i) $\geq 85 \%$ genotypic concordance in the Ag1000G database; (ii) overlap with a restriction enzyme recognition site; (iii) PCR primers fulfilling Primer3Plus default parameters; (iv) clearly distinguishable electrophoretic profiles among inversion genotypes (Table 1, Fig. 1). For simplicity, we named these assays according to the restriction enzyme employed: Cac8I, BstUI, HaeII, and HinfI.

Note that owing to distinct filtering requirements among molecular approaches, two of the four tag SNPs targeted by these assays differ from those employed in the respective sets of 23 and 11 tags developed for highthroughput amplicon sequencing and array hybridization genotyping of $2 \mathrm{R} c$ [15] (see Table 1), though the common principles underlying all $2 \mathrm{R} c$ tags suggest that their performance should be similar.

\section{Validation in the Burkina Faso sample}

We previously genotyped $2 \mathrm{R} c$ in a sample of $463 \mathrm{An}$. coluzzii collected from Burkina Faso, using three independent methods: cytogenetic karyotyping, highthroughput amplicon sequencing, and TaqMan array hybridization (see Methods). Three-way concordance among the methods exceeded $94 \%$, and two-way agreement between the molecular approaches was even higher (97.7\%) despite the fact that only two tag SNPs were shared between them [15]. Genomic DNA remaining from specimens subjected to this three-fold genotyping was employed in the validation of the four PCR-RFLP assays. We defined the reference $2 \mathrm{R} c$ genotype for each specimen-the 'gold standard' against which PCR-RFLP assays were compared-as the consensus genotype indicated by at least two of the three previous methods (Additional file 1: Table S1). Aggregate and individual PCR-RFLP assay genotypes were compared against this reference $2 \mathrm{R} c$ genotype. The aggregate PCR-RFLP assay genotype was defined by majority rule, i.e. at least three of four PCR-RFLP assays agreed. There were 26 of 463 specimens $(5.6 \%)$ for which no aggregate genotype could be defined, either because two different genotypes were each supported by two assays or three different genotypes were supported among the four (Additional file 1: Table S1). Of the remaining 437 specimens that could be assigned aggregate genotypes, all matched the corresponding reference $2 \mathrm{R} c$ genotype (Table 2; Additional file 1: Table S1).

Not surprisingly, the concordance between individual PCR-RFLP assays and the reference $2 \mathrm{R} c$ genotype was imperfect, varying from $\sim 80 \%$ to $\sim 97 \%$ (Table 2). The most important factor underlying incomplete congruence is simply that none of the tag SNPs detected by PCR-RFLP assays are deterministic for inversion orientation in the Ag1000G variation database, presumably owing to low levels of recombination and gene conversion between opposite orientations in heterozygotes (see Ref. 36). As such, no single assay will unerringly predict the correct inversion genotype. Moreover, the percent concordance between tag and chromosomal arrangement 
Table 1 PCR-RFLP genotyping assays for inversion 2Rc in An. coluzzii

\begin{tabular}{|c|c|c|c|c|c|c|c|}
\hline Tag position (breakpoints) & Concord & Ref/Alt & RE & Chr cut & Primer Pairs $\left(5^{\prime} \rightarrow 3^{\prime}\right)$ & Amplicon (bp) & $\begin{array}{l}\text { Cleavage } \\
\text { (bp) }\end{array}$ \\
\hline \multicolumn{8}{|l|}{$(2 \mathrm{R}: 2675000)$} \\
\hline $2 R: 27283425$ & $89.5 \%$ & $\mathrm{G} / \mathrm{A}$ & Cac8l & $2 R+{ }^{c}$ & $\begin{array}{l}\text { F: TAATCGTGTTCGTTCGGTCA } \\
\text { R: CCGGGTACACCTGGAAAGTA }\end{array}$ & 239 & 125,114 \\
\hline 2R:28121178 & $87.2 \%$ & $\mathrm{C} / \mathrm{T}$ & BstUl & $2 \mathrm{R}+{ }^{\mathrm{c}}$ & $\begin{array}{l}\text { F: AAATGCGTGTTGCACTTGAC } \\
\text { R: CCAATAAATCCTAACCCACACG }\end{array}$ & 224 & 173,51 \\
\hline $2 R: 30386671^{a}$ & $86.9 \%$ & $G / A$ & Haell & $2 R+{ }^{c}$ & $\begin{array}{l}\text { F: GGAAAGTTCACGAGCCAAAA } \\
\text { R:TGAGCTTTAGCGACTGCAAG }\end{array}$ & 270 & 165,105 \\
\hline $2 R: 30392408^{b}$ & $94.1 \%$ & $A / G$ & Hinfl & $2 R c$ & $\begin{array}{l}\text { F: AGCTGCCAGGATTTTGTACG } \\
\text { R: CGGCGGGGAAAGTAATTTAT }\end{array}$ & 233 & 136,97 \\
\hline
\end{tabular}

$(2 \mathrm{R}: 31473100)$

Tag position (breakpoints): chromosome coordinates of tag SNP and estimated breakpoint positions from Sangaré [35]; Concord: minimum percent concordance of tag with inversion genotype in Ag1000G based on Love et al. [15]; Ref/Alt, reference and alternate allele at tag SNP; RE, restriction enzyme; chr cut, chromosome (inverted or standard) expected to be cleaved in the assay

a Tag SNP also employed in both high-throughput genotyping panels as described in Love et al. [15]

b Tag SNP also employed in the amplicon sequencing genotyping panel as described in Love et al. [15]

observed in different population samples is expected to vary at least somewhat from the value observed in the Ag1000G database, for stochastic reasons alone, if not due to temporal, geographic, or other population genetic differences. Additional (non-exclusive) more minor sources of disagreement between the reference genotype and the genotype predicted by PCR-RFLP assays include SNP variation in the enzyme recognition site at positions other than the tag itself, SNP variation in the primer binding sites on one or both chromosomal arrangements that reduce or preclude primer binding (often referred to as 'allelic dropout' and typically recognized as a heterozygote deficit), and technical problems with restriction digestion (partial or complete failure of the restriction enzyme to cleave an intact target site).

The two assays that were least concordant with the reference genotype in the Burkina Faso sample were BstUI
(80.3\%) and HaeII (88.6\%). In neither case did we find evidence for significant heterozygote deficits or any striking imbalance in the distribution of discordances across genotypes. In fact, the HaeII assay in our sample slightly outperformed its predicted genotypic concordance (87\% based on the Ag1000G database; Table 1), suggesting that this factor alone is sufficient to explain the HaeII assay's performance. The BstUI assay, by contrast, underperformed its predicted genotypic concordance in Ag1000G (87\%; Table 1). We sequenced a subset of $19 \mathrm{BstUI}$ amplicons from specimens whose PCR-RFLP assay disagreed with the reference genotype (Additional file 1: Table S1). We identified three cases in which an additional SNP destroyed the BstUI restriction target site and abrogated cleavage (despite the allelic state of the tag SNP matching the reference genotype in all three cases), which at least

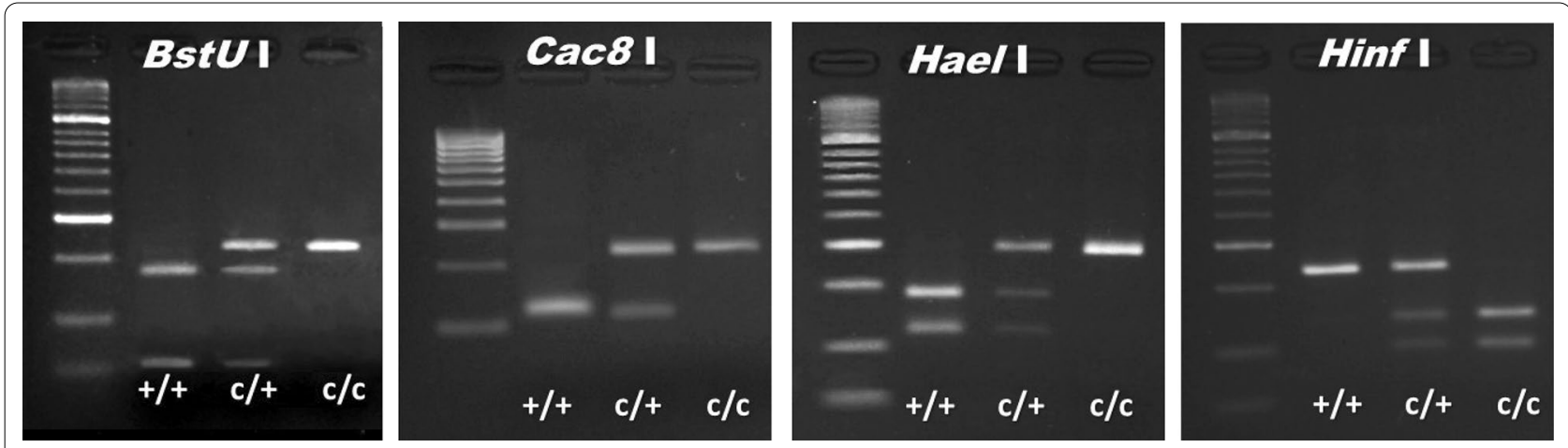

Fig. 1 Representative electrophoretic profiles of the BstUI, Cac8l, Haell, and Hinfl assays for inversion genotyping of 2Rc. Standard (uninverted) homozygotes for 2Rc, +/+; heterozygotes, c/+; inverted homozyogtes, c/c. Molecular weight markers (Bioline; Memphis, TN, USA) are the 50-bp ladder for BstUI, Haell, and Hinfl assays and the 100-bp ladder for Cac8l 


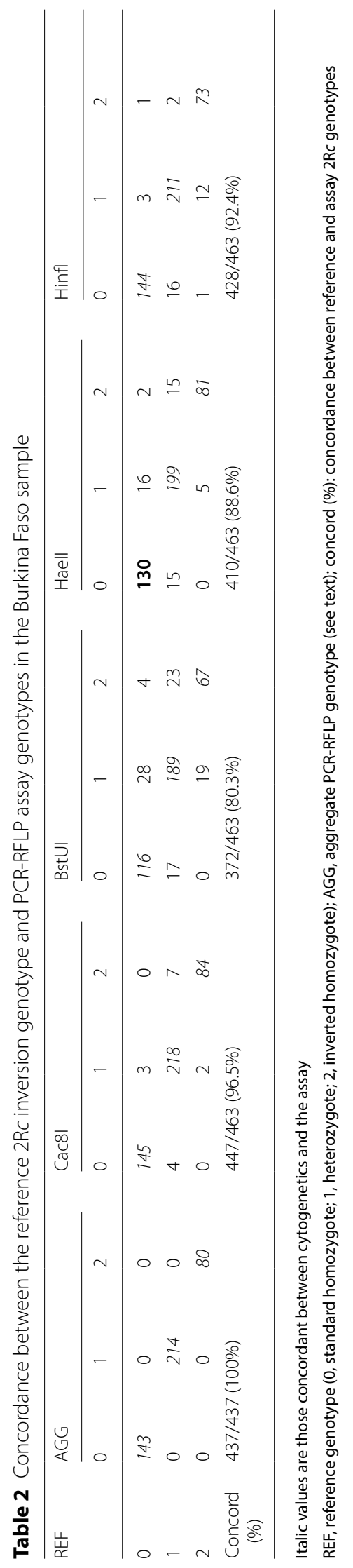


partly explains the apparent underperformance of this assay.

The remaining two assays, Cac8I and HinfI, agreed more often with the reference genotype $(96.5 \%$ and $92.4 \%$, respectively; Table 2). The Cac8I assay performed considerably better in the Burkina Faso sample than predicted based on the genotypic concordance of the tag in Ag1000G (89.5\%; Table 1). Of the 16 specimens with discrepant genotypes, we sequenced the Cac8I amplicons of 12 (Additional file 1: Table S1), finding three whose discrepancies were explained not by the allelic state of the tag SNP (which agreed with the reference genotype in all three specimens) but by a different SNP that destroyed the restriction target site. The performance of Hinfl closely matched expectations based on its tag in Ag1000G (94.1\%; Table 1); we did not perform sequencing on Hinfl amplicons from Burkina Faso owing to COVID-19 restrictions, but we have some insight based on sequencing data from other population samples (see below).

\section{Validation in other population samples}

To extend our analysis spatially, we analyzed samples from four additional countries in West and Central Africa (Mali, Senegal, Benin, Cameroon) that had been subject to cytogenetic analysis, but not high-throughput molecular genotyping approaches. For these specimens, the reference $2 \mathrm{R} c$ genotype was based solely on cytogenetics, although this method is not without human error (up to $4 \%$ or more, depending upon degree of training and experience; $[16,18])$. We compared the cytogenetic genotype to the aggregate (majority rule) and individual PCR-RFLP assay genotypes in pooled (Table 3) and individual (Additional file 2: Table S2) population samples. Due to small sample size and lack of $2 \mathrm{R} c$ inversion polymorphism in some individual population samples, we present below the results based on pooled samples. Similar to the Burkina Faso population sample, we found nearly perfect concordance (98\%) between cytogenetics and the aggregate genotype (184 of 187), after excluding nine specimens whose four PCR-RFLP assays did not produce a majority genotype. Among the three discrepancies (Table 3), one specimen showed a ' 0 ' karyotype (i.e. homozygous standard, confirmed cytogenetically) contradicted by three of four PCR-RFLP assays showing a genotype of ' 1 ' (i.e. heterozygote, with the Bst $U$ I gentotypes confirmed by sequencing). The other two specimens showed a ' 1 ' karyotype, contradicted by all four PCR-RFLP assays indicating a ' 0 ' genotype. In both specimens, an inversion loop in the $2 \mathrm{R} c$ region was confirmed cytogenetically but, due to the relatively low quality of the polytene chromosomes, it was not possible to rule out that the loop corresponds to a rare inversion in the same chromosomal region [28].

Qualitatively, the performance of individual PCR-RFLP assays was comparable inside and outside of Burkina Faso. The same two assays with concordances $<90 \%$ in Burkina Faso (BstUI and HaeII) also performed below $90 \%$ elsewhere (Table 3). Considering that the concordance of their tag SNPs was $\sim 87 \%$ in Ag1000G, these assays actually met expectations. However, the higher correlation between the tag SNPs of the other two assays (Cac8I and HinfI) and inversion status in Ag1000G make them better prospective candidates than BstUI and HaeII. Indeed, in agreement with results from Burkina Faso, both Cac8I and HinfI were superior at genotyping elsewhere in West and Central Africa. Although the Cac8I assay in these other samples did not match its 96.5\% performance in Burkina Faso, it was nevertheless concordant with cytogenetics $>91 \%$ of the time. Sequencing the PCR amplicons of a subset of nine specimens with discordances between the Cac8I assay and cytogenetics revealed that in five cases the tag SNP genotype actually agreed with cytogenetics. In two of those cases, the PCR-RFLP assay disagreement was caused by a different SNP that destroyed the restriction site. Similarly, the HinfI assay was $~ 93 \%$ concordant with cytogenetics in these same samples. Sequencing of 11 PCR amplicons from specimens with genotypic discordances between the assay and cytogenetics revealed no additional polymorphisms in the HinfI recognition site.

Our previous efforts to develop tag SNPs for inversion genotyping were directed toward maximizing geographic and taxonomic inclusion based on An. coluzzii and An. gambiae samples represented in Ag1000G at the time [16]. For three inversions $(2 \mathrm{~L} a, 2 \mathrm{R} b$, and $2 \mathrm{R} u)$, a single set of tags was identified that successfully genotyped both sister species $[15,16]$. By contrast, population structure between An. coluzzii and An. gambiae in the $2 R j, 2 R d$, and $2 R c$ arrangements dictated the development of taxon-specific tags [15, 16]. For 2R $j$ and $2 \mathrm{R} d$, the tags are specific for An. gambiae and are not applicable in An. coluzzii. In the case of inversion $2 \mathrm{R} c$, separate tag sets were successfully developed for in silico genotyping of both taxa [16]. However, application of these tags for high-throughput molecular genotyping of Burkina Faso samples (independent of Ag1000G) revealed that only An. coluzzii tags performed faithfully against cytogenetically karyotyped An. coluzzii specimens; An. gambiae tags applied to An. gambiae specimens were inadequate [15]. Hence, in the present work, using these same Burkina Faso samples (and others), we focused our PCR-RFLP assay development exclusively on An. coluzzii. The reasons for heterogeneous tag performance across different taxa and 
Table 3 Concordance between the cytogenetic 2Rc inversion genotype and PCR-RFLP assay genotypes in pooled samples from Senegal, Mali, Benin, and Cameroon

\begin{tabular}{|c|c|c|c|c|c|c|c|c|c|c|c|c|c|c|c|}
\hline \multirow[t]{2}{*}{$\overline{C Y T}$} & \multicolumn{3}{|c|}{ AGG } & \multicolumn{3}{|c|}{ Cac8l } & \multicolumn{3}{|c|}{ BstUI } & \multicolumn{3}{|c|}{ Haell } & \multicolumn{3}{|c|}{ Hinfl } \\
\hline & 0 & 1 & 2 & 0 & 1 & 2 & 0 & 1 & 2 & 0 & 1 & 2 & $\overline{0}$ & 1 & 2 \\
\hline 0 & 88 & 1 & 0 & 86 & 6 & 2 & 82 & 9 & 3 & 70 & 22 & 2 & 91 & 3 & 0 \\
\hline 1 & 2 & 62 & & 4 & 58 & 3 & 5 & 59 & 1 & 4 & 57 & 4 & 3 & 62 & 0 \\
\hline 2 & 0 & 0 & 34 & 0 & 2 & 35 & 2 & 3 & 32 & 0 & 4 & 33 & 0 & 8 & 29 \\
\hline \multicolumn{4}{|c|}{$\begin{array}{l}\text { Concord 184/187 (98.4\%) } \\
(\%)\end{array}$} & \multicolumn{3}{|c|}{ 179/196 (91.3\%) } & \multicolumn{3}{|c|}{ 173/196 (88.3\%) } & \multicolumn{3}{|c|}{ 160/196 (81.6\%) } & \multicolumn{3}{|c|}{$182 / 196(92.9 \%)$} \\
\hline
\end{tabular}

Italic values are those concordant between cytogenetics and the assay

CYT, cytogenetic genotype ( 0 , standard homozygote; 1 , heterozygote; 2 , inverted homozygote); AGG, aggregate PCR-RFLP genotype (see text); concord (\%), concordance between CYT and assay 2Rc genotypes

even among population samples of the same taxon have yet to be examined in detail, but are likely caused by population structure, which uncouples the correlation between a tag SNP and inversion orientation. Possible (non-exclusive) sources of population structure, aside from taxonomic boundaries themselves, include geography, different selective regimes on allelic targets within inversions, and/or different molecular origins of the inversion. With respect to the last factor, indirect evidence based on allelic variation near the $2 \mathrm{R} c$ breakpoints is consistent with the idea of multiple origins [16], although computational haplotype phasing or long molecule sequencing leading to molecular breakpoint characterization will be required for a confident resolution. Inversion $2 \mathrm{R} c$ is also peculiar in that it is almost never found alone on chromosome $2 \mathrm{R}$. Instead, $2 \mathrm{R} c$ is in nearly perfect linkage disequilibrium with the inverted arrangement of either $2 \mathrm{R} b$ (i.e. $2 \mathrm{R} b c$ ) or $2 \mathrm{R} u$ (i.e. $2 \mathrm{R} c u$ ) $[10,16]$. Of note, $c u$ is a characteristic SAVANNA karyotypic arrangement common in many populations of $A n$. gambiae (s.s.), while $b c$ is a characteristic MOPTI karyotypic arrangement that predominates in arid populations of $A n$. coluzzii. Except for a highly endemic chromosomal form of An. gambiae (s.s.) known as BAMAKO [10, 11], An. gambiae carriers of the $c u$ arrangement are underrepresented in the Ag1000G database. Future development of 2Rc tags and genotyping assays in non-BAMAKO An. gambiae may benefit from additional whole-genome sequencing of An. gambiae carriers of $c u$.

Several factors, most importantly the non-deterministic nature of tag SNPs predictive of $2 \mathrm{R} c$ genotype, operate to prevent any single tag-and any single PCR-RFLP assay dependent on that tag-from unerringly predicting the correct inversion genotype. However, we have shown here that the joint application of multiple PCR-RFLP assays targeting different tags can substantially improve genotypic concordance. For laboratories unwilling or unable to invest in high-throughput genotyping, the most efficient strategy for accurate genotyping of $2 \mathrm{R} c$ which minimizes 'false positives' at the cost of some 'false negatives', would be to apply both the Cac8I and HinfI assays jointly to each specimen in a population sample, preserving only those specimens with genotypes supported by both assays. Had this approach been adopted in the Burkina Faso sample of 463 An. coluzzii, 414 specimens (89.4\%) would have had concordant Cac8I and HinfI assay genotypes. Of those 414, all genotypes except one $(99.8 \%)$ would have agreed with the reference $2 \mathrm{R} c$ genotype. From the original sample of 463, 49 specimens (10.6\%) would have been excluded because of conflicting PCR-RFLP assay genotypes.

There are several limitations of any PCR-RFLP approach. First, and arguably most important, this method is premised on a naturally occurring restriction site overlapping the tag SNP of interest, severely limiting the choice of amenable tag SNPs. Second, the process requires two steps, hence more time: PCR, followed by restriction digestion. Third, restriction enzymes may be costly, difficult to obtain commercially, and labile even if handled carefully. Finally, genotyping errors result both from technical failures of restriction digestion even if the cut site is intact, and from additional polymorphisms arising in the restriction enzyme recognition sequence that destroy the site. We recommend the consistent use of positive controls as indicators of successful restriction enzyme activity. However, beyond this best practice, the other limitations remain. Overcoming these limitations requires the development of a genotyping assay that dispenses with the need for restriction digestion of the amplicon. Quite recently, a rapid and inexpensive approach termed "SuperSelective (SS) PCR" was developed to genotype single nucleotide variants in Caenorhabditis elegans directly following endpoint PCR [37]. This approach has broad application in any genetic system including An. coluzzii. Moreover, it can be developed 
for any tag, irrespective of overlap with a restriction enzyme target site, as it avoids the RFLP step entirely and depends only on specific PCR detection of the SNP itself. Accordingly, to overcome the limitations of PCRRFLP for inversion genotyping, future efforts should be directed at optimizing this SS approach, focusing on those tag SNPs with the highest levels of genotypic concordance in Ag1000G.

\section{Conclusions}

Although improved technological approaches can be anticipated in the future, we have developed two serviceable PCR-RFLP assays, Cac8I and HinfI, that, when used in tandem, allow highly accurate genotyping of inversion $2 \mathrm{R} c$ in $A n$. coluzzii without the need for specialized equipment or training. Because they are rapid and inexpensive, they can be applied in field settings. These assays provide invaluable tools to investigate the association between $2 \mathrm{R} c$ and ecologically or epidemiologically relevant phenotypes and advance our understanding of their molecular basis, aiding our understanding of the evolutionary diversification of this important malaria vector.

\section{Supplementary Information}

The online version contains supplementary material available at https://doi. org/10.1186/s13071-021-04657-x.

Additional file 1. Table S1. Total sample metadata and inversion genotypes for $2 R C$

Additional file 2. Table S2. Concordance between the cytogenetic 2Rc inversion genotype and PCR-RFLP assay genotypes in samples from Senegal, Mali, Benin, and Cameroon.

\section{Acknowledgements}

We thank the village chiefs and all inhabitants of the sampled villages in Burkina Faso, Mali, Senegal, Benin, and Cameroon who allowed collection of mosquitoes from their houses and the local entomological technicians in those countries for their fundamental contributions to the field work. We thank Vincenzo Petrarca for sharing his An. gambiae collections and expertise and M. Kern for technical assistance.

\section{Authors' contributions}

NJB and ADT designed research; RMG, ACV, MC, and VP performed research; RRL, MWG, RKD, MP, CC, and FS contributed reagents/analytic tools; RMG, ACV MC, and VP analyzed data; RMG and NJB wrote the paper. All authors read and approved the final manuscript.

\section{Funding}

This study was supported by the US National Institutes of Health (R01 Al125360). During this work, NJB was supported by the Bill \& Melinda Gates Foundation (Target Malaria, OPP1141988). Under the grant conditions of the Foundation, a Creative Commons Attribution 4.0 Generic License has already been assigned to the Author Accepted Manuscript version that might arise from this submission. ADT was supported by Progetti di Ricerca Università SAPIENZA 2018. ACV received summer support from the Glynn Family Honors Program of the University of Notre Dame. RMG was supported in part by a Kinesis-Fernández Richards Family Fellowship from the University of Notre Dame.

\section{Availability of data and materials}

Data supporting the conclusions of this article are included in the article and its supplementary files. Sequences determined from this study are available in GenBank under accession numbers MW158473-MW158543.

\section{Declarations}

Ethics approval and consent to participate

Not applicable.

\section{Consent for publication}

Not applicable.

\section{Competing interests}

The authors declare that they have no competing interests.

\section{Author details}

${ }^{1}$ Department of Biological Sciences, University of Notre Dame, Notre Dame, IN 46556, USA. ${ }^{2}$ Eck Institute for Global Health, University of Notre Dame, Notre Dame, IN 46556, USA. ${ }^{3}$ Dipartimento di Sanità Pubblica e Malattie Infettive, Università "La Sapienza", Istituto Pasteur-Fondazione Cenci-Bolognetti, 00185 Rome, Italy. ${ }^{4}$ Centre National de Recherche et Formation sur Le Paludisme (CNRFP), Ouagadougou, Burkina Faso. ${ }^{5}$ Institut de Recherche en Sciences de la Santé (IRSS)/Centre Muraz, Bobo-Dioulasso, Burkina Faso. ${ }^{6}$ MIVEGEC, University of Montpellier, IRD, CNRS, Montpellier, France.

Received: 13 January 2021 Accepted: 23 February 2021

Published online: 22 March 2021

\section{References}

1. Kirkpatrick M. How and why chromosome inversions evolve. PLoS Biol. 2010;8:e1000501.

2. Hoffmann AA, Rieseberg LH. Revisiting the impact of inversions in evolution: from population genetic markers to drivers of adaptive shifts and speciation? Annu Rev Ecol Evol Syst. 2008;39:21-42.

3. Wellenreuther M, Bernatchez L. Eco-evolutionary genomics of chromosomal inversions. Trends Ecol Evol. 2018;33:427-40.

4. Kirkpatrick M, Barton N. Chromosome inversions, local adaptation and speciation. Genetics. 2006;173:419-34.

5. Thawornwattana Y, Dalquen D, Yang Z. Coalescent analysis of phylogenomic data confidently resolves the species relationships in the Anopheles gambiae species complex. Mol Biol Evol. 2018;35:2512-27.

6. Coetzee M, Hunt RH, Wilkerson R, della Torre A, Coulibaly MB, Besansky NJ. Anopheles coluzzii and Anopheles amharicus, new members of the Anopheles gambiae complex. Zootaxa. 2013;3619:246-74.

7. Coluzzi M, Sabatini A, Petrarca V, Di Deco MA. Chromosomal differentiation and adaptation to human environments in the Anopheles gambiae complex. Trans R Soc Trop Med Hyg. 1979;73:483-97.

8. White BJ, Collins FH, Besansky NJ. Evolution of Anopheles gambiae in relation to humans and malaria. Annu Rev Ecol Evol Syst. 2011;42:111-32.

9. Barron MG, Paupy C, Rahola N, Akone-Ella O, Ngangue MF, Wilson-Bahun TA, et al. A new species in the major malaria vector complex sheds light on reticulated species evolution. Sci Rep. 2019;9:14753.

10. Coluzzi M, Sabatini A, della Torre A, Di Deco MA, Petrarca V. A polytene chromosome analysis of the Anopheles gambiae species complex. Science. 2002;298:1415-8.

11. Toure YT, Petrarca V, Traore SF, Coulibaly A, Maiga HM, Sankare O, et al. The distribution and inversion polymorphism of chromosomally recognized taxa of the Anopheles gambiae complex in Mali. West Africa Parassitologia. 1998;40:477-511.

12. Scott JA, Brogdon WG, Collins FH. Identification of single specimens of the Anopheles gambiae complex by the polymerase chain reaction. Am J Trop Med Hyg. 1993;49:520-9.

13. DellaTorre A, Fanello C, Akogbeto M, Dossouyovo J, Favia G, Petrarca V, et al. Molecular evidence of incipient speciation within Anopheles gambiae s.s. in West Africa. Insect Mol Biol. 2001;10:9-18. 
14. Coluzzi M. Heterogeneities of the malaria vectorial system in tropical Africa and their significance in malaria epidemiology and control. Bull WHO. 1984;62(Suppl):107-13.

15. Love RR, Pombi M, Guelbeogo MW, Campbell NR, Stephens MT, Dabire $\mathrm{RK}$, et al. Inversion genotyping in the Anopheles gambiae complex using high-throughput array and sequencing platforms. G3 (Bethesda). 2020;10:3299-307.

16. Love RR, Redmond SN, Pombi M, Caputo B, Petrarca V, della Torre A, et al. In silico karyotyping of chromosomally polymorphic malaria mosquitoes in the Anopheles gambiae complex. G3 (Bethesda). 2019;9:3249-62.

17. Lukindu M, Love RR, Guelbeogo MW, Small ST, Stephens MT, Campbell $N R$, et al. High-throughput genotyping of common chromosomal inversions in the Afrotropical malaria mosquito Anopheles funestus. Insects. 2020;11:693.

18. Montanez-Gonzalez R, Pichler V, Calzetta M, Love RR, Vallera A, Schaecher $L$, et al. Highly specific PCR-RFLP assays for karyotyping the widespread $2 \mathrm{Rb}$ inversion in malaria vectors of the Anopheles gambiae complex. Parasit Vectors. 2020;13:16

19. Costantini C, Ayala D, Guelbeogo WM, Pombi M, Some CY, Bassole IHN, et al. Living at the edge: biogeographic patterns of habitat segregation conform to speciation by niche expansion in Anopheles gambiae. BMC Ecol. 2009;9:16.

20. Petrarca V, Vercruysse J, Coluzzi M. Observations on the Anopheles gambiae complex in the Senegal River Basin. West Africa Med Vet Entomol. 1987;1:303-12.

21. Akogbeto M, Di Deco MA. Polytene chromosome study on Anopheles gambiae in Benin and Togo. West Africa J of African Zoology. 1995;5-6:443-54.

22. Simard F, Ayala D, Kamdem GC, Etouna J, Ose K, Fotsing J-M, et al. Ecological niche partitioning between the $\mathrm{M}$ and $\mathrm{S}$ molecular forms of Anopheles gambiae in Cameroon: the ecological side of speciation. BMC Ecol. 2009;9:17.

23. Santolamazza F, Mancini E, Simard F, Qi Y, Tu Z, della Torre A. Insertion polymorphisms of SINE200 retrotransposons within speciation islands of Anopheles gambiae molecular forms. Malar J. 2008;7:163.

24. Fanello C, Santolamazza F, della Torre A. Simultaneous identification of species and molecular forms of the Anopheles gambiae complex by PCRRFLP. Med Vet Entomol. 2002;16:461-4.

25. Santolamazza F, Della Torre A, Caccone A. Short report: A new polymerase chain reaction-restriction fragment length polymorphism method to identify Anopheles arabiensis from An. gambiae and its two molecular forms from degraded DNA templates or museum samples. Am J Trop Med Hyg. 2004;70:604-6.

26. della Torre A. Polytene chromosome preparation from anopheline mosquitoes. In: Crampton JM, Beard CB, Louis C, eds. Molecular biology of disease vectors: a methods manual. London: Chapman \& Hall; 1997. p. 329-36.

27. George P, Sharakhova MV, Sharakhov IV. High-resolution cytogenetic map for the African malaria vector Anopheles gambiae. Insect Mol Biol. 2010;19:675-82.

28. Pombi M, Caputo B, Simard F, Di Deco MA, Coluzzi M, della Torre A, et al. Chromosomal plasticity and evolutionary potential in the malaria vector Anopheles gambiae sensu stricto: insights from three decades of rare paracentric inversions. BMC Evol Biol. 2008;8:309.

29. Miles A, Harding NJ, Bottà G, Clarkson CS, Antão T, Kozak K, et al. Genetic diversity of the African malaria vector Anopheles gambiae. Nature. 2017:552:96-100

30. Anopheles gambiae Genomes C. Genome variation and population structure among 1142 mosquitoes of the African malaria vector species Anopheles gambiae and Anopheles coluzzii. Genome Res. 2020;30:1533-46.

31. Ma J, Amos Cl. Investigation of inversion polymorphisms in the human genome using principal components analysis. PLoS ONE. 2012;7:e40224.

32. Vincze T, Posfai J, Roberts RJ. NEBcutter: a program to cleave DNA with restriction enzymes. Nucleic Acids Res. 2003;31:3688-91.

33. Giraldo-Calderon Gl, Emrich SJ, MacCallum RM, Maslen G, Dialynas E, Topalis P, et al. VectorBase: an updated bioinformatics resource for invertebrate vectors and other organisms related with human diseases. Nucleic Acids Res. 2015;43:D707-13.

34. Untergasser A, Cutcutache I, Koressaar T, Ye J, Faircloth BC, Remm M, et al. Primer3-new capabilities and interfaces. Nucleic Acids Res. 2012;40:e115.

35. Sangare DM: Breakpoint analysis of the Anopheles gambiae s.S. chromosome 2Rb, 2Rc, and 2Ru inversions. Notre Dame, IN: University of Notre Dame; 2007.

36. Cheng C, White BJ, Kamdem C, Mockaitis K, Costantini C, Hahn MW, et al. Ecological genomics of Anopheles gambiae along a latitudinal cline: a population-resequencing approach. Genetics. 2012;190:1417-32.

37. Touroutine D, Tanis JE. A rapid, superselective method for detection of single nucleotide variants in Caenorhabditis elegans. Genetics. 2020;216:343-52.

\section{Publisher's Note}

Springer Nature remains neutral with regard to jurisdictional claims in published maps and institutional affiliations.
Ready to submit your research? Choose BMC and benefit from:

- fast, convenient online submission

- thorough peer review by experienced researchers in your field

- rapid publication on acceptance

- support for research data, including large and complex data types

- gold Open Access which fosters wider collaboration and increased citations

- maximum visibility for your research: over $100 \mathrm{M}$ website views per year

At BMC, research is always in progress.

Learn more biomedcentral.com/submissions 